\title{
Quantitative Evaluation of Stress-Strain Curves by Spherical-Tip Nanoindentation with Variable Radius Method*
}

\author{
Iwao MATSUYA**, Randy Gui Guan FATT** and Ikuo IHARA** \\ ** Department of Mechanical Engineering, Nagaoka University of Technology, \\ 1603-1 Kamitomioka, Nagaoka, Niigata 940-2188, Japan \\ E-mail: ihara@mech.nagaokaut.ac.jp
}

\begin{abstract}
We propose a novel evaluation method to obtain precise stress-strain curves by spherical-tip nanoindentation with continuous multiple loading technique. The small-sized spherical-tip indenters have an imperfect shape and it causes miss-fitting with tensile test result. We adopted the variable radius method, where we defined the precise indenter radius as the function of contact depth to calculate the stress-strain response precisely. We calculated the representative stress and representative strain by combining Hollomon's law, Hill's model, proper strain model, and the variable radius and compared with the tensile test result. It is found that stress-strain curves obtained by using the variable radius method agree well with the tensile test result rather than using nominal radius. These results clearly indicate the effectiveness of our method to evaluate the stress-strain response by using the small-sized spherical-tip indenter.
\end{abstract}

Key words: Spherical-Tip Nanoindentation, Stress-Strain Curve, Variable Radius Method, Work Hardening Exponent, Strength Coefficient

\section{Introduction}

In the fields of science and engineering, there are growing demands for measuring materials properties of small-sized materials and thin films used for MEMS (Micro Electro Mechanical Systems) as well as many other industrial products. In particular, mechanical properties which are directly related to the elastic and plastic behaviors of materials are strongly required to be measured because accurate values of such properties are indispensable to ensure higher reliability in making better machine design and CAE applications. Measuring mechanical properties of materials in submilimeter or submicrometer volumes using conventional techniques such as uniaxial tensile tests and Micro Vickers hardness tests can be difficult. Thus, there are surprisingly little knowledge about material properties of such volumes even for widely used industrial materials such as steel and alloys. Spherical-Tip nanoindentation with continuous multiple loading indentation technique has been established as a sophisticated technique capable of obtaining stress-strain response of materials involving material surface of a small volume ${ }^{(1),(2)}$. These material properties obtained by nanoindentation can then be used as representative values for plastic and elastic properties in machine designing, CAE analyses and material science researches. However, current works provide stress-strain curves that are reliable only at post-yielding region while a micrometer-sized spherical-tip indenter can be used to measure an even more localized area $^{(3)}$. In addition small-sized spherical-tip indenter has an imperfect shape which has a direct influence on indentation surface area calculation of 
materials $^{(4),(5)}$.

In this paper, to examine such influences due to the imperfection of the tip shape, we focused on the actual indenter shape, which affects the calculation results. We adopted the variable radius method, where we defined the precise indenter radius as the function of indentation depth to calculate the stress-strain response precisely. The obtained stress-strain curves of steel are then compared with the tensile test result. The proper analysis conditions that provide results correlating closely with tensile test result are then presented and discussed.

\section{Determining Stress and Strain Relationship}

When a spherical indenter penetrates into a material as shown in Fig. 1, the deformation is classified into three stages of elastic, elastic plastic, and fully plastic. When the stress field due to indentation satisfies the yield condition, local plastic deformation starts to occur beneath the spherical indenter and expands to the free surface. The mean contact pressure gradually increases in the fully plastic zone. This deformation behavior in indentation is similar to uniaxial tensile test. When the indenter proceeds to penetrate, the so called "pile-up" or "sink in" occurs at the edge of the indenter which leads to change in contact area for a given depth. Figure 1 represents a "pile-up" situation where $R$ is the radius of spherical tip, $a$ is contact radius and $h_{c}$ is contact depth with consideration to piling-up, $a$ ' and $h_{c}{ }^{\prime}$ is without consideration to piling up.

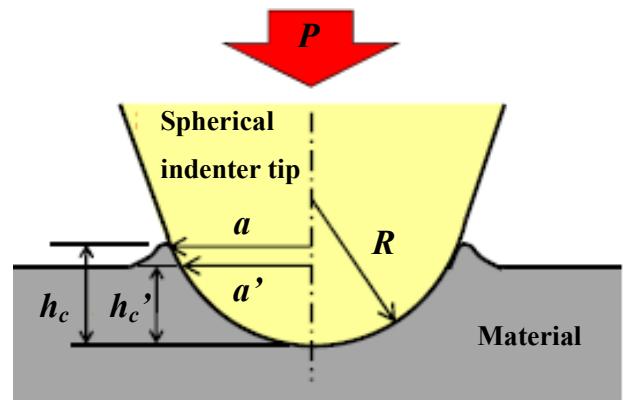

Figure 1. Schematic diagram of spherical-tip nanoindentation.

Representative stress $\sigma_{R}$ can be expressed using mean contact pressure $P_{m}$ as shown below,

$$
\sigma_{R}=\frac{P_{m}}{c}=\frac{P}{c \pi a^{2}}
$$

where $P$ is maximum terminal load at each unloading step, $c$ is constraint factor depending on deformation regime. For simplicity, we use $c=2.8$, which corresponds to fully plastic regime. The value of $a$ is determined using model of Hill et al. as follows ${ }^{(6)}$.

$$
\begin{aligned}
& a=\sqrt{2 R h_{c}-h_{c}{ }^{2}} \\
& h_{c}=\frac{5}{2} \cdot \frac{(2-n)}{(4+n)} \cdot h_{c}^{\prime}
\end{aligned}
$$

This model is acceptable for dimensionless contact radii, $a / R$, higher than 0.3 , which matches the parameter condition of our experiment using the continuous multiple loading technique ${ }^{(7)}$. Here, $n$ is work hardening exponent or strain hardening exponent.

The value of $n$ can be calculated using an iteration method. Using an initial value of $n=$ 0.3 , we can obtain stress-strain relation from representative stress and representative strain models. The value $n$ is then recalculated using the Hollomon's law as shown below,

$$
\sigma_{R}=K \varepsilon_{R}^{n}
$$


where $K$ is strength coefficient, and $\varepsilon_{R}$ is representative strain. This procedure is then iterated until the input value equals the return value. In this work, representative strain model is defined as the average plastic strain depth in direction by analyzing the area change during indentation ${ }^{(4),(8)}$

$$
\varepsilon_{R}=\ln \frac{2}{1+\cos \gamma}=\ln \frac{2}{1+\sqrt{1-(a / R)^{2}}}
$$

Here, $\gamma$ is the contact angle of the indenter and specimen. The work hardening exponent obtained through Hollomon's equation is then compared to those obtained from uniaxial tensile test.

\section{Experimental Method and Calculation Procedure}

Test specimens used were alloy steel (SM490 JIS standard). The specimen's dimension for indentation tests was about $5 \times 5 \times 1 \mathrm{~mm}$. The specimen surface was ground and polished with $1 \mu \mathrm{m} \mathrm{Al}{ }_{2} \mathrm{O}_{3}$ powders. A load-controlled indenter ENT-2100 from Elionix Inc., Japan, is employed. This tester provides continuous multiple loading/partial-unloading indentations with load and displacement resolutions of $4.0 \mathrm{nN}$ and $0.06 \mathrm{~nm}$, respectively ${ }^{(9)}$. The relationship between indentation load and penetration depth were measured continuously by using diamond spherical-tip indenters with radius of $5 \mu \mathrm{m}, 10 \mu \mathrm{m}$, and 15 $\mu \mathrm{m}$. Indentation was repeated at 7 different locations.

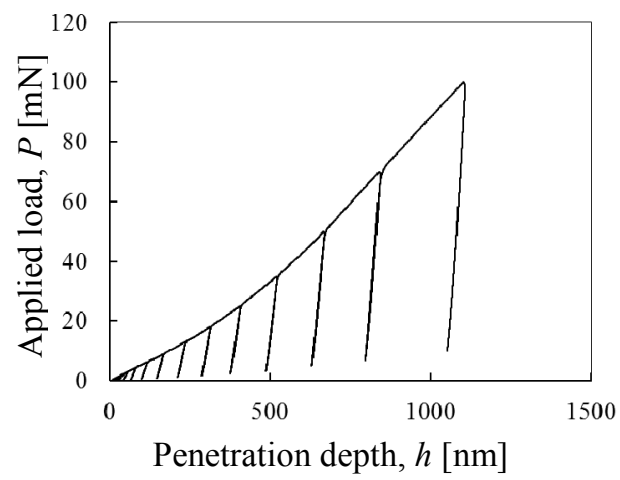

(a)

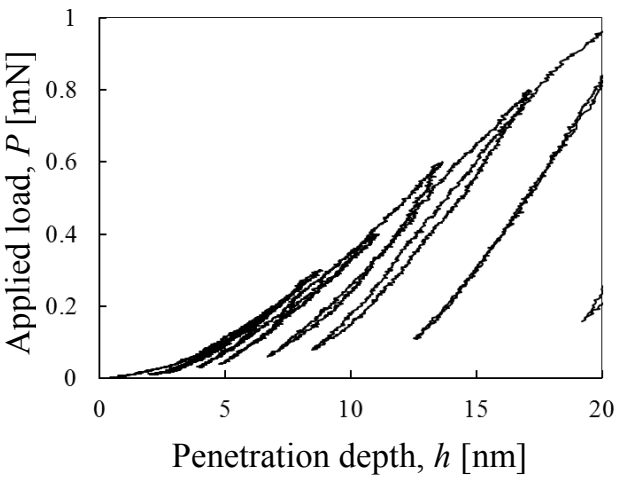

(b)

Figure 2. Typical load-depth diagram for continuous multiple loading, (a) the whole, (b) the detail at the beginning of loading.

Figure 2 shows a typical measurement result of continuous multiple indentations load versus indentation depth for a steel specimen. The specimen is indented with a series of 21 loading/unloading sequences with increasing terminal load from $0.1 \mathrm{mN}$ to $100 \mathrm{mN}$ as shown in Fig. 2(a). To account for thermal drift in this method, the load is removed not totally but partially at $10 \%$ of terminal load, reloading and unloading are then repeated, as shown in Fig. 2(b) until the final maximum load at $100 \mathrm{mN}$ is reached. Since both representative stress and strain can be determined from loading/unloading curve at each step, stress-strain curve is then given as a set of 21 data points.

We observed the radius of the indenter tip by using SEM (Scanning Electron Microscope) to investigate the precise tip-radius according to the contact depth and defined the variable radius by the function of contact depth. SEM observation of indenter was performed with more than 5000 magnification. Obtained SEM images are binarized to obtain the profiles of indenters. The spatial resolution of the binarized image is about 30 $\mathrm{nm}$. Based on the binarized image, the radius of the indenter tip is determined quantitatively through the following procedure. At first, the original point is set on the front edge of 
indenter, and by drawing the line perpendicular to the direction of indentation at a certain penetration depth, two intersection points of the line and the indenter profile which is a contour of the binarized image are determined. Then the profile of the indenter tip is approximated by a circular arc which is determined from the three points, the original point and two intersection points. The same procedure is performed for different penetration depth of the indenter at every $100 \mathrm{~nm}$ until the maximum indentation depth and the relation between the radius of the approximated circular arc and the contact depth is obtained. Finally, the radius of the approximated circular arcs is expressed as a one or two-dimensional function of the contact depth.

We calculated the stress and strain relationship by combining variable radius method, Hollomon's law ${ }^{(10)}$, proper strain model ${ }^{(4),(11)}$. In addition we used the strength coefficient $K$ in Eq. (4) with constant value of $990 \mathrm{MPa}$ which was obtained from tensile test because we wanted to examine the effectiveness of variable radius method by getting rid of extraneous factor. Obtained data were then converted to stress-strain curves and compared with tensile test result. It is more reasonable to be able to estimate not only the work hardening exponent $n$ but also the strength coefficient $K$ for unknown materials. In an optimization problem to find the two unknown coefficients, $n$ and $K$, weighting factors used for determining these unknown coefficients are quite important and their appropriate values are indispensable. However, it is rather difficult to explore the optimum weighting factor at this stage and is necessary to make further investigation. To follow up this matter would carry us too far away from the main subject in this work, therefore we simply use the strength coefficient $K$ determined from the tensile test of the steel and the work hardening exponents were estimated by the iteration method mentioned above in chapter 2 .

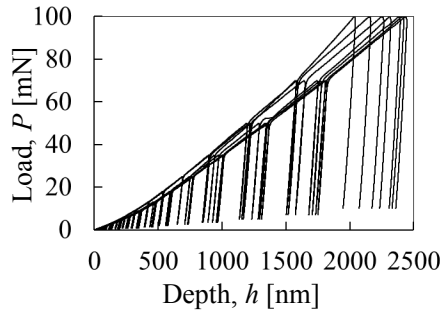

(a)

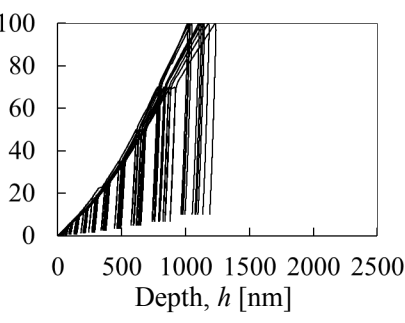

(b)

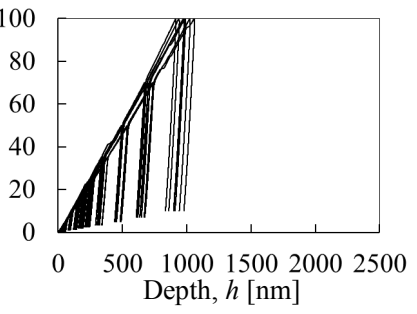

(c)

Figure 3. Load-depth diagrams obtained by continuous multiple loading method with (a) 5 $\mu \mathrm{m}$, (b) $10 \mu \mathrm{m}$, and (c) $15 \mu \mathrm{m}$ indenter tip.

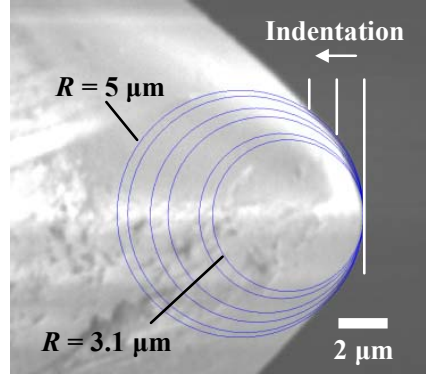

(a)

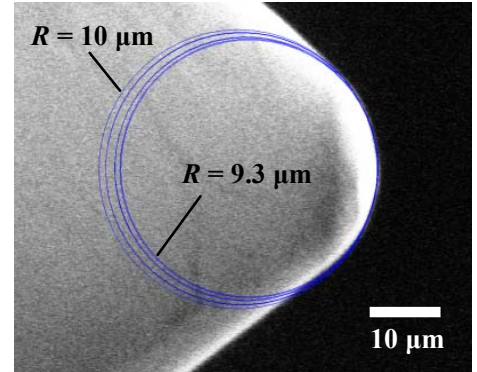

(b)

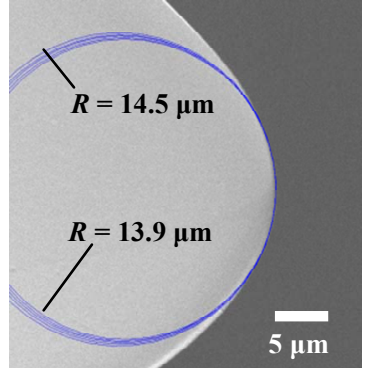

(c)

Figure 4. SEM image of the spherical-tip indenters. Nominal radius of (a) $5 \mu \mathrm{m}$, (b) $10 \mu \mathrm{m}$, and (c) $15 \mu \mathrm{m}$. 


\section{Results and Discussion}

Figure 3 shows load-depth diagrams obtained by continuous multiple loading method with nominal radius of 5, 10, and $15 \mu \mathrm{m}$ respectively. As shown in Fig. 3 (a), $5 \mu \mathrm{m}$ results have larger deviation than the others. We can obtain information of penetration depth from these results, and then calculate contact depth by using Eq. (2) and (3).

Figure 4 shows the SEM image of the spherical-tip indenter with nominal radius of 5 $\mu \mathrm{m}, 10 \mu \mathrm{m}$, and $15 \mu \mathrm{m}$. The blue line shows the inscribed circle according to the contact depth $h_{c}$ at each penetration depth. There's big difference between nominal radius and actual shape especially in nominal radius of $5 \mu \mathrm{m}$ indenter. In the previous results, stress and strain curves by using $5 \mu \mathrm{m}$ indenter does not agree well with tensile test result because the nominal radius of $5 \mu \mathrm{m}$ indenter shows the worst shape compared to other indenters. As shown in Fig. 4 (a), the radius of $5 \mu \mathrm{m}$ indenter changes from 3.1 to $5 \mu \mathrm{m}$ with increasing contact depth up to around $2.5 \mu \mathrm{m}$. With the $10 \mu \mathrm{m}$ tip, the radius of the indenter changes from 9.3 to $10 \mu \mathrm{m}$ with increasing indentation depth up to around $1.2 \mu \mathrm{m}$ as shown in Fig. 4 (b). With the $15 \mu \mathrm{m}$ tip, the radius of the indenter changes from 13.9 to $14.5 \mu \mathrm{m}$ with increasing indentation depth up to around $1.0 \mu \mathrm{m}$ as shown in Fig. 4 (c). This result indicates that we have to correct the indenter radius according to the contact depth to calculate the stress-strain relationship correctly because if we use the nominal radius for indenters, the calculation results of stress and strain would be significantly different from true values. The variable radius consisting of the function of contact depth for each indenters are shown as follows,

$$
\begin{aligned}
& R_{5}=-1.086 \times 10^{-4} h_{c}{ }^{2}+1.191 h_{c}+2331.3 \\
& R_{10}=1.191 h_{c}+8903 \\
& R_{15}=-0.6604 h_{c}+14635
\end{aligned}
$$

where $R_{5}$ is the variable radius for nominal radius of $5 \mu \mathrm{m}$ indenter, $R_{10}$ is the variable radius for $10 \mu \mathrm{m}$ indenter, $R_{15}$ is the variable radius for $15 \mu \mathrm{m}$ indenter, in unit of $\mathrm{nm}$ respectively. In this approximation, there are mainly two factors affecting the value of the estimated radius. One is caused by the positional accuracy of circular arc arranged on binarized SEM image of the indenter and the other is caused by the distortion of the indenter profile which deviates from a perfect circular arc. The positional accuracy of the approximated circular arc depends on spatial resolution of the binarized image. Because the spatial resolution of the binarized image is high enough as mentioned above, the reproducibility of the circular arc is also good enough at every contact depth so that the fluctuation of the estimated radius of the approximated circular arc was about $2 \%$ at a maximum. It has also been found from a comparison of the polynomial approximation of indenter profile and the circular arcs of the variable radius at a certain penetration depth that the average correlation coefficients between them are $0.99202,0.99934$ and 0.99916 for $R_{5}$, $R_{10}$, and $R_{15}$, respectively. These results show that the variable radius achieve a certain level of approximation quality, although it is not clear how the distortion of the indenter profile influences the results of the estimated stress-strain relationship. This problem needs to be discussed separately in future research. By substituting the variable radius of Eqs. (6) (8) into Eq. (2), instead of using values of nominal radius, we will be able to obtain better stress and strain curves.

Figure 5 shows the stress-strain curves calculated by using the values of nominal radius and Fig. 6 shows the stress-strain curves calculated by variable radius method, with nominal radius of (a) $5 \mu \mathrm{m}$, (b) $10 \mu \mathrm{m}$, and (c) $15 \mu \mathrm{m}$, respectively. The solid line shows the tensile test result and the white circles show the calculated representative stress and representative strain curves. As shown in Fig. 5, stress-strain curves obtained by $5 \mu \mathrm{m}$ indenter do not agree well with the tensile test result, while results using 10 and $15 \mu \mathrm{m}$ indenter agree better, 
because of the indenter shape. However, as shown in Fig. 6, we can see significant improvement on the difference between nanoindentation and tensile test. These results clearly show that the calculated stress-strain curves by using the variable radius method agree well with the tensile test result rather than using nominal radius.

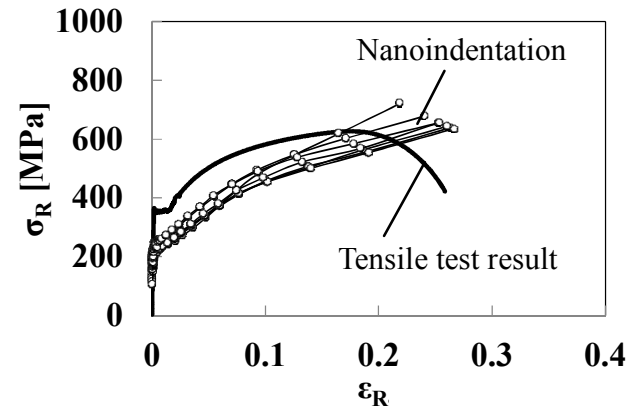

(a)

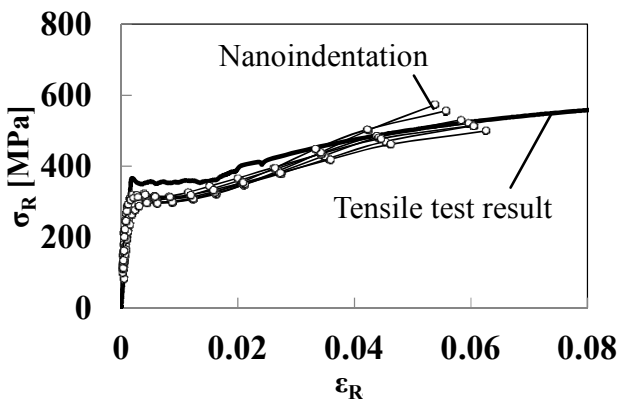

(b)

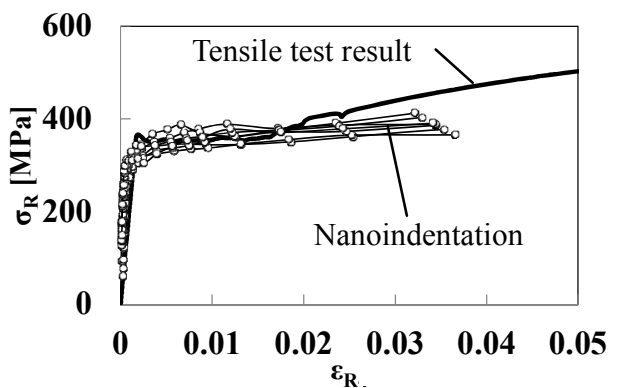

(c)

Figure 5. Stress-Strain curves calculated by using the values of nominal radius. (a) 5 $\mu \mathrm{m}$, (b) $10 \mu \mathrm{m}$, and (c) $15 \mu \mathrm{m}$ indenter tip.

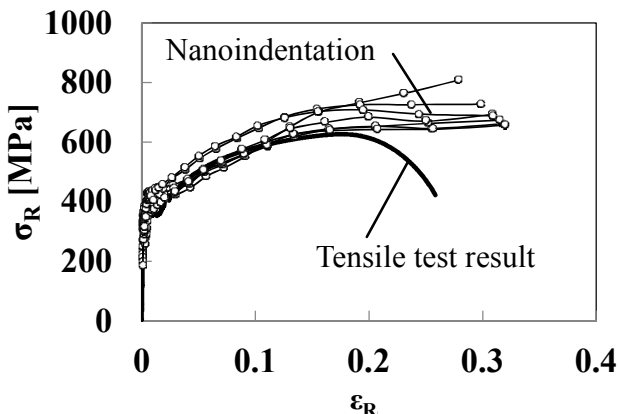

(a)

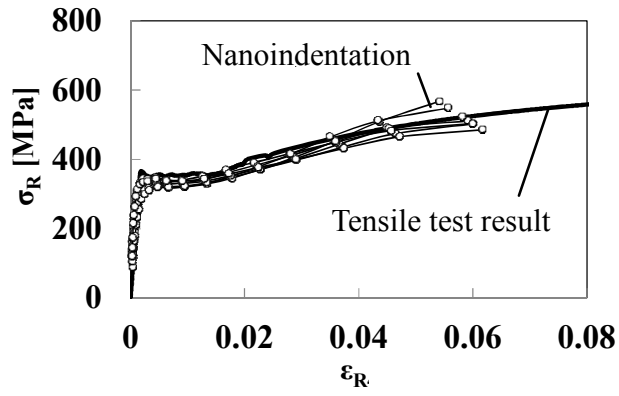

(b)

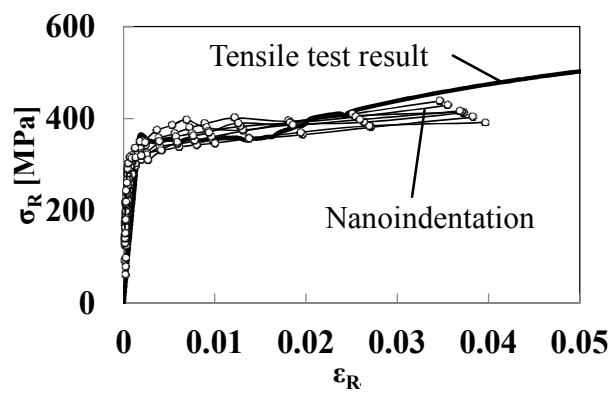

(c)

Figure 6. Stress-Strain curves calculated by variable radius method. (a) $5 \mu \mathrm{m}$, (b) 10 $\mu \mathrm{m}$, and (c) $15 \mu \mathrm{m}$ indenter tip.

Figure 7 shows the values of work hardening exponent $n$ calculated by using the indenters of $5 \mu \mathrm{m}, 10 \mu \mathrm{m}$, and $15 \mu \mathrm{m}$ in nominal radius. Data points plotted in Fig. 7 (a) are calculated by using nominal radius value, and that in Fig. 7 (b) are calculated by using variable radius method. The solid line shows the value of work hardening exponent obtained by tensile test, which is $0.225^{(12)}$. It can be seen from the comparison between Fig. 7 (a) and (b) that the work hardening exponents determined by the continuous multiple loading indentation with the variable radius show no radius dependence, while the results determined with nominal radius show a clear radius dependence. It is noted that the value of $n>0.2857$ exhibits sink-in effect and $n<0.2857$ exhibits pile-up effect on indentation impression on specimen. The threshold value can be derived from Eq. (3) when the penetration depth $h_{c}$ ' equals the contact depth $h_{c}$. It is interesting that the average work 
hardening exponents obtained by the nanoindentation are $0.222,0.245$, and 0.249 for $R_{5}$, $R_{10}$, and $R_{15}$, respectively, which almost agree with that obtained from a tensile test and show the value exhibiting a pile-up effect. Although further study such as a simultaneous determination of the strength coefficient and the work hardening exponent in the present procedure is necessary, the variable radius method developed in this work could be an effective way for evaluating an appropriate stress-strain relationship.

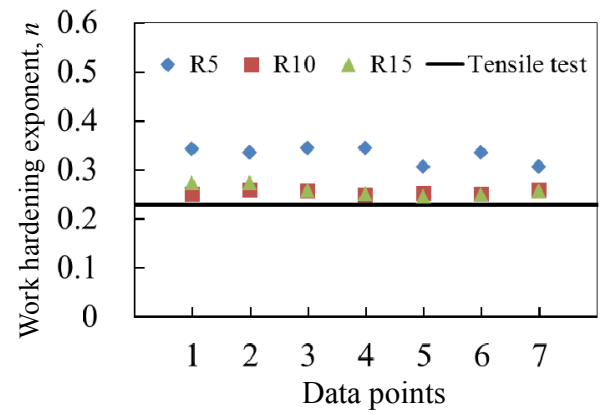

(a)

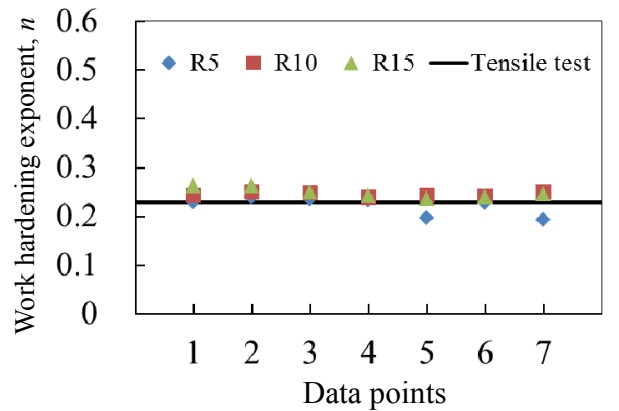

(b)

Figure 7. Work hardening exponent values. (a) Using nominal radius, (b) using the variable radius model, with nominal radius of $5 \mu \mathrm{m}, 10 \mu \mathrm{m}$, and $15 \mu \mathrm{m}$, respectively.

\section{Conclusions}

Stress-strain curves at local area obtained by spherical-tip nanoindentation with continuous multiple loading technique on steel was evaluated. It is shown that the small-sized spherical-tip indenters have an imperfect shape and it causes miss-fitting with tensile test result. We calculated the representative stress and representative strain by combining Hollomon's law, Hill's model, proper strain model, and the variable radius which is the function of contact depth obtained by SEM observation. It is found that stress-strain curves obtained by using the variable radius method agree well with the tensile test result rather than using nominal radius. These results clearly indicate that the novel quantitative method to evaluate the stress-strain information at an even more localized area by using the small-sized spherical-tip indenter is developed.

\section{Acknowledgement}

This research was supported by Elionix Inc., Japan and Grants-in-Aid for Scientific Research (B) Grant Number 22360304.

\section{References}

(1) Oliver, W. C. and Pharr, G. M., An improved technique for determining hardness and elastic modulus using load and displacement sensing indentation experiments, Journal of Material Research, Vol. 7, No.6 (1992), pp.1564-1583.

(2) Oliver, W. C. and Pharr, G. M., Measurement of hardness and elastic modulus by instrumented indentation: Advances in understanding and refinements to methodology, Journal of Material Research, Vol. 19, No.1 (2004), pp.3-20.

(3) Bell, T. J., Bendeli, A., Field, J. S., Swain, M. V. and Thwaite, E. G., The determination of surface plastic and elastic properties by ultra-micro indentation, Metrologia, Vol.28 (1991), pp.463-469.

(4) Fatt, R. G. G., Ihara, I., Yudate, T., Ichimura, M., Uegaki, J. and Shima, Y., Microscopic determination of stress-strain relationship by continuous multiple nano-indentation technique, Proceedings of the 4th JSME/ASME 2011 International Conference on 
Materials and Processing, (2011), pp.51147.

(5) Matsuya, I., Fatt, R. G. G. and Ihara, I., Quantitative evaluation of stress-strain curves at local area by spherical-tip nanoindentation, Proceedings of the 3rd Asian Symposium on Materials and Processing, (2012), pp.NDTC5.

(6) Hill, R., Storakers, B. and Zdunek, A. B., A theoretical study of the Brinell hardness test, Proceedings of the Royal Society of London, Series A, Vol.423, No.1865 (1989), pp.301330.

(7) Bartier, O., Hernot, X. and Mauvoisin, G., Theoretical and experimental analysis of contact radius for spherical indentation, Mechanics of Materials, Vol.42, No.6 (2010), pp.640-656.

(8) Milman, Yu. V., Galanov, B. A. and Chugunova, S. I., Plasticity characteristic obtained through hardness measurement, Acta Metallurgica et Materialia, Vol.41, No.9 (1993), pp.2523-2532.

(9) Ihara, I., Matsumoto, T., Yajima, Y., Uegaki, J. and Shima, Y., Application of continuous multiple loading with spherical-tip nanoindentation to mechanical property evaluations of industrial materials, Proceedings of the IMEKO 2010 TC3, TC5 and TC22 Conferences Metrology in Modern Context, (2010), pp.201-204.

(10) Hollomon, J. H., Tensile deformation, Transactions of the American Institute of Mining and Metallurgical Engineers, Vol.162 (1945), pp.268-290.

(11) Ahn, J. H. and Kwon, D., Derivation of plastic stress-strain relationship from ball indentations: Examination of strain definition and pileup effect, Journal of Materials Research, Vol.16 (2001), pp.3170-3178.

(12) Kamaya, M., Effect of plastic strain on fracture strength of cracked components, INSS journal, Vol.16 (2009), pp.179-188. 